\title{
Typicality Effects and the Logic of Reciprocity
}

\author{
Nir Kerem Naama Friedmann Yoad Winter \\ Technion/ Tel Aviv University Utrecht University/ \\ Google Technion
}

\section{Introduction}

The variability in the interpretation of reciprocal expressions has been extensively addressed in the literature and received detailed semantic accounts. After pointing out a central empirical limitation of previous logical accounts of reciprocity, we argue that these approaches suffer from inadequacies due to ignoring typicality preferences with binary predicate concepts. We claim that typicality preferences are crucial for interpreting reciprocals and introduce a new principle, the Maximal Typicality Hypothesis (MTH), which analyzes reciprocals using an extension of the Strongest Meaning Hypothesis (SMH) proposed in Dalrymple et al. (1998) Unlike the $\mathrm{SMH}$, which is a principle that implicitly presupposes a classical two-valued ("definitional") treatment of predicate concepts, the MTH respects the fuzziness of such concepts as manifested by their typicality preferences, and expects strong correlations between these preferences and the range of logical interpretations available for reciprocal expressions. The expected correlations are supported by new empirical results elicited in a series of experiments with speakers of Hebrew.

Section 2 discusses the SMH and a major challenge for it. Section 3 briefly reviews previous work on typicality in the theory of concepts. Section 4 introduces the MTH and its formalization. Section 5 discusses the empirical settings we used for testing the MTH and introduces our experimental results and their analysis. Section 6 analyzes these results and their implication for the MTH.

\section{Reciprocal interpretation}

One of the central problems in analyzing reciprocals stems from the fact that they do not seem to make a unitary logical contribution to sentences in which they appear. Consider for example the following sentences.

(1) Larry, Monty and Garfield know each other.

(2) Larry, Monty and Garfield are following each other into the room.

All drawings used in the experiments reported in this paper are by Ruth Noy Shapira. We are grateful to Michal Biran for help with the experimental work. We also thank the audiences at SALT XIX, Nijmegen Institute for Cognition and Information, and New York University for their remarks. This work was made possible by grants from the Israel Science Foundation (grant 2005231) and the Netherlands Organization for Scientific Research (NWO), which are gratefully acknowledged. 
The configurations in which Larry, Monty and Garfield may be knowing/ following each other in these two sentences are not the same. While (1) requires that each of the three men knows the two other men, sentence (2) only requires a linear configuration, e.g. one in which Larry is entering the room first, with Monty following him and Garfield following Monty. In order to account for such variations in the interpretation of reciprocal sentences, Dalrymple et al. (1998) catalogue the different meanings reciprocal expressions may receive and propose a principle called the Strongest Meaning Hypothesis (SMH) that selects between them. Reciprocal meanings are ordered according to their logical strength, where a stronger meaning requires more pairs in the binary relation denoted by the transitive predicate antecedent of the reciprocal. Thus, the reciprocal meaning in (1) is assumed to be stronger than the meaning in (2). The SMH in Dalrymple et al.'s formulation describes the interpretation of a reciprocal expression as the strongest available meaning that is not contradicted by the context. For instance, in (1) the context does not prevent each of the three men from knowing the other two. Hence the reciprocal is assigned the strongest meaning possible, requiring a full graph of acquaintances. By contrast, in (2) it is impossible for each man to be following the other two into the room. Therefore a linear configuration is predicted by the SMH using one of the weaker reciprocal meanings that Dalrymple et al. assume.

Sabato and Winter (2005) (henceforth $S \& W$ ) adopt Dalrymple et al.'s general approach, but argue, following Winter (2001), that it is first of all the meaning of the predicate antecedent of the reciprocal that determines its interpretation. $\mathrm{S} \& \mathrm{~W}$ characterize logical parameters that derive directly from the meaning of binary predicates, and further revise the $\mathrm{SMH}$ for describing the effects of these parameters on the interpretation of the reciprocal.

Strongest Meaning Hypothesis (S\&W's version): A reciprocal expression requires the denotation of its predicate antecedent to be a maximal relation that respects the semantic restrictions on the predicate concept.

In (2) the relation denoted by the binary predicate follow into the room must be acyclic and asymmetric due to the meaning of the concept conveyed by this predicate. The predicate know in (1) involves no comparable restrictions. Thus, maximality in S\&W's version of the SMH requires a complete graph in (1) but a weaker interpretation in (2), as reviewed in more detail in Section 4

The two versions of the SMH by Dalrymple et al. and by S\&W have a common property: they both assume that the interpretation of reciprocals is only sensitive to "classical" (or "definitional") aspects of the meaning of relational concepts like know or follow into the room. For instance, the acyclicity of the latter relation can be treated as a non-logical axiom, or a meaning postulate, which holds true in all possible models. The implicit assumption is that the contextual effects (in Dalrymple et al.'s account) or the semantic properties of the predicate (in S\&W's account) that affect the interpretation of reciprocals are all of this sort.

This approach faces a general empirical problem, illustrated by sentence (3).

Larry, Monty and Garfield are combing each other's hair. 
Sentence (3) allows the interpretation in which each of the three men is combing both the of the others' hair, as depicted in Figure 1 (a) below. However, unlike sentence (1), this interpretation is not the only one available for (3). For example, as supported by our empirical study (see Experiment 2 in Section 5), an interpretation in which each of the men only combs the hair of one of his companions, as in Figure 1(b), is also felicitous for (3).
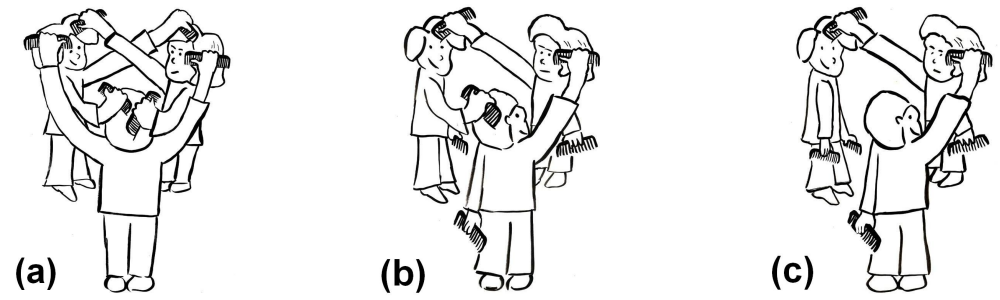

Figure 1: different instances of the relational concept for the verb comb

According to the $\mathrm{SMH}$, the physical possibility that each man combs the hair of the two other men blocks any weaker interpretation for (3), contrary to fact. Interpretations of reciprocal sentences that are weaker than expected by the SMH appear with many other transitive verbs (see Section 5). Our central claim in this paper is that this inadequacy of the SMH results from ignoring meaning properties of binary predicates that involve typicality preferences with natural concepts. With respect to sentence (3), for instance, we propose that the acceptability of the sentence as describing Figure 1.b) is related to the higher typicality of combing one person's hair as a "combing situation" when compared to Figure 1(a). Before presenting our account, however, some background on typicality and its relevance for the semantic theory of concepts is given in the following section.

\section{Concepts and typicality}

Ordinary concepts like FRUIT or BIRD have been the center of much research in Cognitive Psychology, Philosophy, and Linguistics. Many works (see Smith and Medin 1981, Margolis and Laurence 1999 for reviews) have studied the processes in which humans categorize objects as instances of concepts, and developed theories about how these concepts may be represented in the human mind. According to the classical theory of concepts, their representation has a definitional structure expressing the necessary and sufficient conditions for categorizing entities as instances of the concept. A popular example is the concept BACHELOR, which may be defined as the conjunction of the concepts UNMARRIED and MAN. The classical theory assumes that such "definitional" reductions can ultimately describe the mental structure of concepts, as well as their use for categorization.

Despite its influence on the theory of concepts, a central piece of evidence challenging the classical theory involves typicality preferences with categorization. A well-known example of these preferences involves categorizing instances of the 
category BIRD. Instances of birds like robins or sparrows were shown to rate as "better" or "more typical" examples of the concept BIRD than other bird instances like chickens, penguins or ostriches. Instances of the former species are categorized more quickly as birds, acquired earlier in childhood, and listed more often as examples for birds. Some of the categorization tasks that were shown to exhibit such typicality effects include category membership (e.g. Rosch 1975), sentence verification (e.g. Armstrong et al. 1983) and inductive inference (e.g. Osherson 1990), as well as acceptance of qualifying terms (e.g. Lakoff 1973 ), memory encoding and recall (e.g. Rosch 1976, Rosch and Mervis 1975), category learning (e.g. Rosch 1976, Rosch and Mervis 1975) and many other tasks. These works and the correlations they exposed between typicality effects in different categorization tasks, have led to the conclusion that any theory of concepts must take their typicality preferences into account. The classical theory, whatever its status as a theory of concepts may be, takes all instances of a concept as equal members of the category, and therefore does not account for typicality effects in their categorization.

In formalizing typicality, we use Osherson and Smith's (1997) notation, where typicality of entities with respect to a concept CON is described by a typicality function $\Theta_{\text {CoN }}$ that assigns entities values in the interval $[0,1)$ - the non-negative real numbers smaller than 1.1 Elements that are more typical of the concept receive a higher score. For instance, in the above example we assume $\Theta_{\text {BIRD }}\left(\mathbf{r}^{\prime}\right)>\Theta_{\text {BIRD }}\left(\mathbf{p}^{\prime}\right)$, where $\mathbf{r}^{\prime}$ and $\mathbf{p}^{\prime}$ are instances of a robin and a penguin respectively.

\section{The Maximal Typicality Hypothesis}

This section revises and generalizes the $\mathrm{SMH}$, and proposes a modified principle, the Maximal Typicality Hypothesis (MTH), for generating the truth conditions of reciprocal sentences. Instead of only considering the possibility or impossibility of different denotations for binary predicates, as in the $\mathrm{SMH}$, the MTH also takes into account the typicality of different denotations. Revising S\&W's formulation, we informally state the MTH as follows.

Maximal Typicality Hypothesis (MTH): A reciprocal expression requires the denotation of its predicate antecedent to be a relation of maximal typicality relative to the predicate concept.

Intuitively, the idea is that when three people are involved, situations as in Figure 1.b), with each person combing only one other person, are maximally typical for the concept $\mathrm{COMB}$, and hence licensed as situations of reciprocal combing for sentence (3). This is an extension of Dalrymple et al.'s intuition that the linear configuration of people following each other in sentence (2) is a maximal situation for the concept FOLLOW INTO THE ROOM. In the first case, adding pairs to the relation is possible, but atypical; in the latter case, adding pairs to the relation is strictly speak-

\footnotetext{
${ }^{1}$ The reason for not allowing typicality 1 is Osherson and Smith's assumption that no entity is maximally typical as an instance of a concept. Osherson and Smith use the notation ' $c$ ' for the function that we here denote ' $\Theta$ '.
} 
ing impossible. The MTH thus takes the two cases of "incomplete" reciprocity as stemming from the same principles of conceptual structure.

The MTH assumes that similarly to other concepts, concepts underlying binary predicates show typicality preferences between different instances of the predicate. But what is an "instance of the predicate"? With one-place predicate concepts like BIRD, an instance is standardly assumed to be an entity categorized as belonging to the predicate extension. With concepts corresponding to transitive verbs, however, an instance must involve at least two entities, denoted by the subject and the object. Furthermore, we are here also interested in typicality of situations with three or more entities. Consider for example the two situations in Figure 2, The (b) situation is an instance of the predicate comb that only involves one patient. ${ }^{2}$ The (a) situation, however, involves one agent and two patients. To rank the typicality of the two situations as combing exemplars, we assume that it is the denotation of a two-place predicate $c o m b$, restricted to the entities in the situation, that is ranked for typicality. If we standardly assume that transitive verbs like comb denote binary relations, the underlying concept COMB must attribute typicality to different binary relations. In Figure 2 , let $x, y, z$ denote the participating entities. In the (a) situation the restricted denotation of the verb comb is the relation $R_{a}=\{\langle x, y\rangle,\langle x, z\rangle\}$, whereas in (b) it is the relation $R_{b}=\{\langle x, y\rangle\}$. As for the typicality of these relations with respect to the verb concept, we adopt (and later empirically support) the assumption $\Theta_{\text {сомв }}\left(R_{a}\right)<\Theta_{\text {сомв }}\left(R_{b}\right)$.

(a)

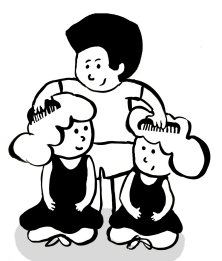

(b)

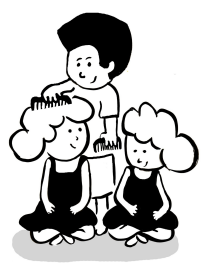

Figure 2: combing with one or two patients

Thus, typicality of binary relations as instances of a concept CON is described using a function $\Theta_{\text {CoN }}$ from binary relations over the domain, elements of $\wp\left(E^{2}\right)$, to typicality values in the interval $[0,1)$. The MTH uses such typicality functions for defining the interpretation of reciprocals. Consider first the semantic structure of sentences like the boys know each other $(=(1))$. The reciprocal expression is analyzed as a relation between sets of entities and binary relations over entities. Accordingly, sentence (1) is analyzed as stating that the reciprocal relation holds between the set of boys and the binary relation denoted by the verb know.

To see how the proposed MTH works, let us first consider S\&W's proposal, which the MTH generalizes. According to S\&W the meaning of a reciprocal expression is defined relative to a semantic restriction describing the possible binary relations instantiating the predicate concept. For instance, in the case of sentence

\footnotetext{
${ }^{2}$ In some of the verbs that we discuss below, the thematic role of the object is not a patient, but a theme. We ignore however this thematic distinction, and consistently use the terms agent and patient for referring to the entity or entities denoted by the subject or the object, respectively.
} 
(2) above, the possible instances of the concept related to the expression follow into the room must all be acyclic and asymmetric relations. Let us denote by $\Theta_{\text {FoLlow }}^{c l}$ such a "classical" semantic restriction, which sends any binary relation to 1 if it is acyclic and asymmetric, and to 0 otherwise. Our notation is meant to highlight our conviction that Dalrymple et al.'s assumption about contextual effects and S\&W's assumption about semantic restrictions should be construed as manifestations of the conceptual structure of two-place predicates, which is more accurately reflected, and formally generalized, using typicality functions.

As said above, S\&W define the operation of the $\mathrm{SMH}$ by analyzing the reciprocal in VPs like follow each other as requiring that the verb (follow) denotes a maximal relation on the set denotation of the subject, relative to the verb's semantic restrictions. In sentence (2), for instance, given the acyclicity and asymmetry of the verb follow, a linear configuration on the three elements of the subject describes a maximal relation for this set: adding any pair to this relation would stand in contradiction to the properties of the predicate concept. More formally, for any domain of entities $E$, a reciprocal expression in S\&W's proposal denotes a relation that holds of the sets $A \subseteq E$ and binary relations $R \subseteq E^{2}$, where $R$ is maximal on $A$ relative to the semantic restriction $\Theta^{c l}$ on the predicate concept (e.g. KNOW, FOLLOW). Maximality of $R$ on $A$ is defined by examining the relation $\left.R\right|_{A}=R \cap A^{2}$. Pairs of binary relations $R_{1}, R_{2}$ should be compared while restricting them to the set $A$ and ignoring identity pairs on $A$ : the pairs $I_{A}=\left\{\langle x, x\rangle \in E^{2}: x \in A\right\}$. The proper equality ('= ${ }_{A}$ ') and containment (' $\subseteq$ ') relations are defined as follows.

$$
\begin{aligned}
& R_{1}={ }_{A} R_{2} \text { iff }\left.R_{1}\right|_{A} \backslash I_{A}=\left.R_{2}\right|_{A} \backslash I_{A} \\
& R_{1} \subseteq{ }_{A} R_{2} \text { iff }\left.\left.R_{1}\right|_{A} \backslash I_{A} \subseteq R_{2}\right|_{A} \backslash I_{A}
\end{aligned}
$$

Informally: when comparing two binary relations $R_{1}$ and $R_{2}$ on a set $A$, we only look at the pairs of elements that they relate within $A$ that are not identity pairs. Ignoring identity pairs is necessary because the meaning of reciprocals is contingent with respect to membership of such pairs in the denotation of the reciprocated binary relation. For instance, the truth of a sentence like John and Bill admire each other does not depend on whether John (or Bill) admires himself or not.

Using these notions of semantic restrictions and equality/containment between binary relations relative to a set $A, \mathrm{~S} \& \mathrm{~W}$ 's definition below applies the $\mathrm{SMH}$ for specifying the meaning of reciprocal relations. ${ }^{3}$

SMH-based reciprocity: Given a semantic restriction $\Theta^{c l}: \wp\left(E^{2}\right) \rightarrow\{0,1\}$, a set of entities $A \subseteq E$ and a binary relation $R \subseteq E^{2}$ s.t. $\Theta^{c l}\left(\left.R\right|_{A}\right)=1$ EXHIBIT RECIPROCITY with respect to $\Theta^{c l}$ if and only if the following holds:

$$
\forall R^{\prime} \subseteq E^{2}: R \subseteq{ }_{A} R^{\prime} \wedge \Theta^{c l}\left(\left.R\right|_{A}\right) \leq \Theta^{c l}\left(\left.R^{\prime}\right|_{A}\right) \Rightarrow R={ }_{A} R^{\prime} .
$$

It is easy to illustrate that under this formulation a reciprocal relation must hold between a set $\{x, y, z\}$ and a linear relation follow ${ }^{\prime}=\{\langle x, y\rangle,\langle y, z\rangle\}$, provided that

\footnotetext{
${ }^{3}$ Our formulation of S\&W's proposal contains here a slight redundancy: due to the assumption $\Theta^{c l}\left(\left.R\right|_{A}\right)=1$, the requirement $\Theta^{c l}\left(\left.R\right|_{A}\right) \leq \Theta^{c l}\left(\left.R^{\prime}\right|_{A}\right)$ boils down to requiring $\Theta^{c l}\left(\left.R^{\prime}\right|_{A}\right)=1$. This redundancy only comes to highlight the generalization embodied in our definition of the MTH below.
} 
the semantic restriction $\Theta_{\text {FOLLow }}^{c l}$ contains all and only the acyclic asymmetric binary relations over the domain. By contrast, we can assume that the semantic restriction $\Theta_{\text {KNOw }}^{c l}$ allows all possible relations in the domain, since there is no a priori restriction on who knows who. Unlike follow, the reciprocal relation would not hold for linear relations denoted by know, as these are not maximal relative to the semantic restriction $\Theta_{\text {KNow }}^{c l}$. This analysis captures the contrast between sentences (1) and (2).

The idea behind the MTH is similar to S\&W's formulation of the SMH, but it takes typicality of binary relations as the core semantic information on binary predicate concepts, rather than "classical" semantic restrictions on their meaning. Reconsider the two situations in Figure 1 . While Figure 1 (b) does not contain a maximal number of possible pairs in the denotation of the predicate comb, we assume that it is a situation of maximal typicality for the concept. Thus, adding more pairs is possible (see Figure 1(a)), but doing this might result in a situation that is less typical of the concept COMB. We hypothesize that this is the reason for the acceptability of the reciprocal sentence (3) in Figure 1(b), contrary to what the SMH expects. Revising S\&W's formulation of the SMH using this notion of maximal typicality, we propose the following MTH-based definition.

MTH-based reciprocity: Given a typicality function $\Theta: \wp\left(E^{2}\right) \rightarrow[0,1)$, a set of entities $A \subseteq E$ and a binary relation $R \subseteq E^{2}$ s.t. $\Theta\left(\left.R\right|_{A}\right)>0$ EXHIBIT RECIPROCITY with respect to $\Theta$ if and only if the following holds:

$$
\forall R^{\prime} \subseteq E^{2}: R \subseteq{ }_{A} R^{\prime} \wedge \Theta\left(\left.R\right|_{A}\right) \leq \Theta\left(\left.R^{\prime}\right|_{A}\right) \Rightarrow R={ }_{A} R^{\prime} .
$$

According to the MTH, thus, if for all pairs $\left\langle a_{1}, a_{2}\right\rangle$ of non-equal elements $a_{1} \neq a_{2}$ in $A$, adding $\left\langle a_{1}, a_{2}\right\rangle$ to $R$ reduces $R$ 's typicality on $A$, then $A$ and $R$ are said to stand in the reciprocity relation. In the two situations of Figure 1 , let us denote the relevant combing relations $R_{a}{ }^{\prime}$ and $R_{b}{ }^{\prime}$ respectively. We assume that the typicality $\Theta_{\text {сомв }}\left(R_{b}{ }^{\prime}\right)$ is higher than the typicality of any situation where a combing action is added to $R_{b}{ }^{\prime}$. If this is the case, then the MTH correctly expects $R_{b}{ }^{\prime}$ to exhibit reciprocity with respect to the three people in the situation. Note that according to the MTH, the complete relation $R_{a}{ }^{\prime}$ also exhibits reciprocity with respect to these three people. This is since, provided that the typicality of $R_{a}{ }^{\prime}$ is bigger than zero, it is maximally typical in the sense of the MTH: adding non-identity pairs to the situation is impossible, and hence trivially cannot reduce its typicality. Even more generally, adding any pair to the three pairs in situation (b) is expected to monotonically reduce the typicality of the situation for the concept сомв. Under this assumption, any of the situations with 4, 5 and 6 combing pairs is expected by the MTH to exhibit reciprocity, as we believe is the case.

Since the MTH is strictly speaking an extension of S\&W's formalization of the SMH, it also extends its results, after moving from "classical" semantic restrictions to more general typicality functions. Specifically, the contrast between the predicates know and follow in sentences (1) and (2) is accounted for in a similar way to S\&W's account. For the predicate know, we may reasonably assume that typicality increases monotonically or remains constant when adding more pairs to the relation. According to this assumption, only a maximal knowing relation, in- 
cluding all possible non-identity pairs, supports sentence (11) according to the MTH. Further, S\&W's assumption that all and only acyclic and asymmetric relations are possible denotations for the predicate follow can be described using a typicality function that sends only such relations to a positive value, whereas cyclic and/or asymmetric relations are assigned typicality zero. With this assumption on typicality for follow, we may again assume that typicality grows monotonically or remains constant when adding pairs to a relations within the set of acyclic asymmetric relations. This accounts for the contrast with the predicate know: adding any pair to a linear configuration would reduce its typicality to zero, whereas taking pairs out of such a configuration would reduce its typicality, or leave it intact. Consequently, according to the $\mathrm{MTH}$, a linear configuration exhibits reciprocity for the predicate follow but not for the predicate know.

Concluding, the MTH encodes the lexical semantic assumptions of the SMH as typicality functions and preserves some desired empirical aspects of S\&W's system. However, because the MTH is a more general principle than the $\mathrm{SMH}$, it is used to account for phenomena that cannot be captured using the classical theory of concepts. In the remainder of this paper, we turn to a preliminary experimental study of the implications of the MTH.

\section{Experimental support for the Maximal Typicality Hypothesis}

The MTH establishes a relation between typicality effects with binary predicates and the interpretation of reciprocal expressions appearing with them. For instance, when comparing Figures 1(a) and 1(b), the MTH assumes that it is the higher typicality of the latter as an instance of the concept COMB, which licenses reciprocity in it. Reasonably, the main factor that makes Figure 1(a) an atypical instance of the concept is the fact that each of the persons in it is the agent, as well as the patient, of two combing activities. Thus, in Figure 2 we expect the typicality of the (b) situation, with one patient, to be higher than the typicality of the (a) situation, where two patients are combed simultaneously by the same agent. Typicality preferences with such "one agent" situations are assumed to reflect the basic conceptual structure of verbs, and to correlate with the typicality of the more complex situations of Figure 1. In the four experiments that we describe below, we make the tentative experimental assumption that typicality preferences in "one agent" situations are indicative of typicality of situations with more agents (and patients). It follows that we expect typicality judgements about patient cardinality in situations like Figures 2 (a) and 2(b) to correlate with reciprocity judgements about situations like Figures 1 (a) and 1 (b) respectively. This expectation was tested in two pairs of experiments presenting situations that were examined for their typicality and reciprocity relative to sentences of natural language. In Experiments 1 and 2 the situations were presented graphically, and in Experiments 3 and 4, they were presented textually. All tests were performed on native speakers of Modern Hebrew, university students from Tel Aviv University and Technion, Israel Institute of Technology. 


\subsection{Experiment 1 - preferences of patient cardinality (picture-based)}

This experiment tested typicality preferences with binary predicates in Hebrew using picture selection in depicted situations that differ in their patient cardinality.

Method 53 participants (39 male and 14 female, average age 24) were given a form containing 38 questions, of which 32 were test items and 6 fillers. Each test item covered one binary concept, and was designed to compare the typicality judgements on two instances of this concept. These instances were illustrated in two drawings, where one agent performs the relevant activity on one patient or on two patients. Apart from the number of patients the two drawings were as similar as possible. In addition to these two drawings, each test item contained a sentence, and the typicality judgement was elicited by introducing to the participants a forced choice question: which of the two depicted situations better describes the sentence? In all items, the subject of the sentence was visibly the agent in the drawings, and the verb corresponding to the binary concept in question appeared without an object. For example, for the binary predicate concept HIT, the two drawings in Figure 3 were presented together with sentence (4)

(a)

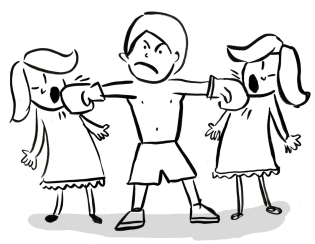

(b)

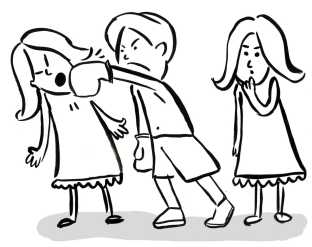

Figure 3: hitting with one or two patients

Filler items contained two pictures that differed from one another in respects other than patient cardinality (e.g location or instrument). In order to cancel out priming effects, the questionnaire forms had two versions in opposite orders of the items. In both versions of the questionnaire, if the same predicate served both for a filler and in a test item, then the test item came before the filler.

Results Different verbs showed different preferences for the cardinality of objects. Some verbs (such as 'stab', 'shake' and 'comb') showed a clear preference for a single object, whereas other verbs (e.g. 'give a speech' and 'take a picture') showed a preference for multiple objects. Yet other verbs showed no significant preference, with choices not significantly different from random ('catch' or 'blind', for example). Table 1 shows the predicates tested and for each predicate, the percentage of participants who preferred the picture depicting one patient over the picture with two patients. 


\begin{tabular}{|c|c|c|c|c|c|}
\hline Predicate & Translation & $\begin{array}{l}\text { Preference for } \\
\text { single patient } \\
\text { (percent) }\end{array}$ & Predicate & Translation & $\begin{array}{l}\text { Preference for } \\
\text { single patient } \\
\text { (percent) }\end{array}$ \\
\hline no'em & 'give a speech' & 7.5 & medagdeg & 'tickle' & 64.2 \\
\hline mecalem & 'photograph' & 24.5 & maSpric & 'spray' & 67.3 \\
\hline mecayer & 'draw' & 26.4 & cove'a & 'paint' & 69.2 \\
\hline mexabek & 'hug' & 32.1 & soret & 'scrape' & 70.6 \\
\hline mena'ane'a & 'rock / lull' & 38.5 & menake & 'clean' & 71.7 \\
\hline medaber & 'talk to' & 41.5 & me'aper & 'apply makeup' & 71.7 \\
\hline Sotef & ‘wash’ & 41.5 & noge'a & 'touch' & 73.1 \\
\hline maSmi'a & 'play [sound]' & 42.0 & yore & 'shoot' & 73.6 \\
\hline mefasel & 'sculpt' & 43.4 & covet & 'pinch' & 74.5 \\
\hline mesanver & 'dazzle' & 50.0 & mesarek & 'comb' & 77.4 \\
\hline roxec & 'bathe' & 50.0 & menagev & 'towel' & 81.1 \\
\hline tofes & 'catch' & 51.9 & macbi'a & 'point at' & 86.5 \\
\hline melatef & 'caress' & 53.8 & make & 'hit' & 86.5 \\
\hline martiv & 'wet' & 56.6 & mena'er & 'shake' & 86.8 \\
\hline doxef & 'push' & 58.5 & doker & ‘stab’ & 88.2 \\
\hline mesaben & 'soap' & 61.5 & & & \\
\hline
\end{tabular}

\subsection{Experiment 2 - preferences of reciprocal interpretations (picture-based)}

Experiment 2 tested acceptance of reciprocal interpretations of sentences containing a subset of the predicates used in Experiment 1. The predicates chosen for this experiment were the predicates that showed highest or lowest preferences for a single patient in Experiment 1, and that most easily allowed graphical representation of the relevant reciprocal situations.

Method Experiment 2 was set up similarly to Experiment 1. 50 participants (30 male and 20 female, average age 25) were given a form consisting of 49 questions: 26 test items and 23 fillers. Each test item contained two drawings and a reciprocal sentence with a subject referring to three people. The two pictures were as similar as possible, each depicting three people acting on each other in a different constellation. As in Experiment 1, the participants were asked to choose which of the two pictures better describes the sentence. For each verb, the questionnaire included two test items. In one test item for the verb, one of the pictures depicted six pairs of activities between the human figures (i.e. every figure acting on each of the two other figures), and the other picture depicted three pairs (each figure acting on the figure to its right). In the two pictures of the other test item for the verb, one picture depicted three pairs identically to the first test item, and the other picture depicted two pairs of activities, where the agents in the two pairs were two different figures. The reciprocity judgement was elicited by asking the participants a forced choice question: which of the two depicted situations better describes the sentence? For example, for the binary predicate concept COMB, the two test items consisted of Figure 1 (a) compared to Figure 1 (b), and of Figure 1.(b) compared to Figure 1 (c), 
each pair accompanied by the following reciprocal sentence:

$$
\begin{aligned}
& \text { danny, gai ve'omer mesarkim ze-et-ze } \\
& \text { Danny, Guy and-Omer comb each other } \\
& \text { "Danny, Guy and Omer are combing each other." }
\end{aligned}
$$

The filler items presented non-reciprocal sentences and pictures.

Results Table 2 shows the predicates tested and, for each predicate, the percentage of participants who preferred the three-pair picture to the six-pair picture, as well as the percentage preferring the two-pair picture to the three-pair picture.

\begin{tabular}{llll}
\hline Predicate & Translation & \multicolumn{2}{c}{ Participants' preferred picture (percent) } \\
& & 3 pairs over 6 & 2 pairs over 3 \\
\hline mexabek & 'hug' & 10.0 & 2.0 \\
make & 'hit' & 18.4 & 4.0 \\
covea & 'paint' & 24.0 & 0.0 \\
macbi'a & 'point at' & 28.6 & 0.0 \\
covet & 'pinch' & 34.0 & 2.0 \\
mena'er & 'shake' & 34.0 & 2.0 \\
doker & 'stab' & 38.0 & 6.0 \\
menake & 'clean' & 40.0 & 2.0 \\
menagev & 'towel' & 40.0 & 6.1 \\
me'aper & 'apply makeup' & 40.0 & 6.0 \\
soret & 'scrape' & 42.0 & 6.0 \\
no'em & 'give a speech' & 42.0 & 6.0 \\
mesarek & 'comb' & 65.3 & 6.0 \\
\hline
\end{tabular}

Table 2: reciprocal interpretations preferred in Experiment 2

From these results we see that verbs like 'comb', which show a preference for a single patient, do not require complete configurations for reciprocal sentences in which they appear. By contrast, all verbs, independently on their preferences for patient cardinality, strongly prefer configurations with 3 pairs to 2 pairs as situations supporting reciprocal sentences.

\subsection{Experiment 3 - preferences of patient cardinality (text-based)}

Similarly to Experiment 1, Experiment 3 tested typicality preferences with binary predicates in Hebrew using elicited judgements on situations that differ in their patient cardinality. Unlike Experiment 1, here the situations were verbally described, and in each test item the participants were asked to choose between a singular object a plural object in a transitive sentence illustrating the concept in question.

Method 50 (41 male and 9 female, average age 25) participants were given a form containing 28 questions: 18 test items and 10 fillers. Each test item consisted of an incomplete transitive sentence in which the object was missing. The incomplete sentence was accompanied by two possible objects, one singular and one plural. 
Participants were asked to choose which of the two objects completed the sentence in a way that sounds best to them. In this textual experiment, unlike the graphical Experiment 1, it was experimentally easier to use verbs for referring to activities in the past. We hypothesize that the aspect of the activity, especially whether it was completed (perfective aspect) or not (imperfective aspect), may affect the typicality of the activity. Eventive predicates like 'to compliment' were tested in both the perfective and the imperfective. Stative verbs like 'hate', were only tested in the perfective aspect. When two test items contained the same verb, at least three other test items separated between them. Modern Hebrew lacks overt aspectual marking, and the aspect (perfective/imperfective) of the activity was highlighted using the tense of the sentence (past/present, respectively) and the use of report verbs with the imperfective. Sentences (6) and (7) illustrate the test items in the perfective and imperfective aspect, respectively, for the verb maxmi ('compliment').

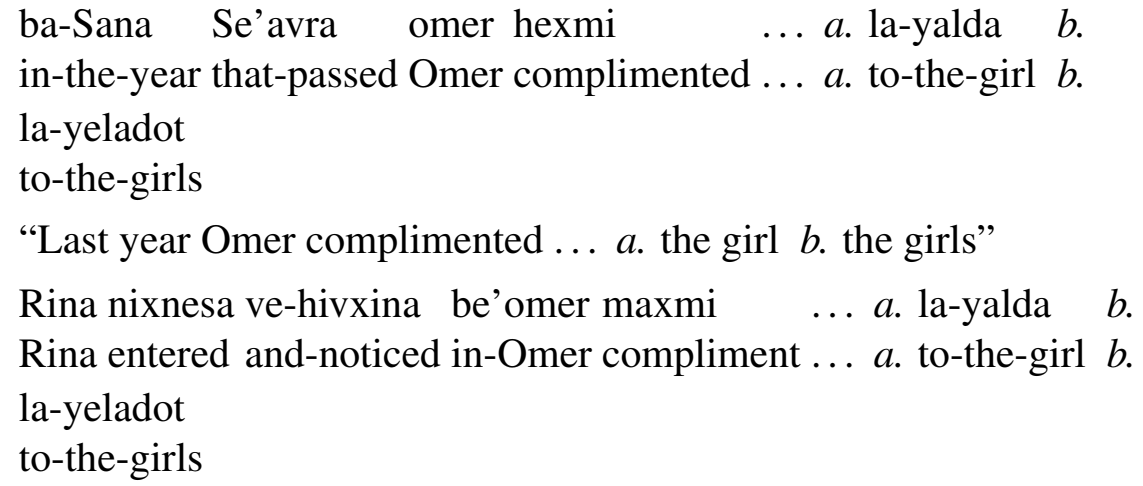

The filler items contained objects that differed from one another in respects other than patient cardinality (e.g. gender).

Results Table 3 shows for each predicate predicate, in its possible aspects, the percentage of participants who preferred the singular object to the plural object.

\begin{tabular}{|c|c|c|c|}
\hline \multirow[t]{2}{*}{ Predicate } & \multirow[t]{2}{*}{ Translation } & \multicolumn{2}{|c|}{ Preference for singular object (percent) } \\
\hline & & perfective & imperfective \\
\hline no'em & 'give a speech' & 10.4 & 14.0 \\
\hline ro'e & 'see' & 25.0 & 63.3 \\
\hline makSiv & 'listen to' & 42.9 & 66.7 \\
\hline maxmi & 'compliment' & 62.0 & 77.1 \\
\hline macbia & 'point at' & 81.3 & 72.9 \\
\hline soret & 'scrape’ & 93.8 & 95.8 \\
\hline doker & 'stab' & 95.8 & 86.0 \\
\hline mefake'ax & 'supervise' & 18.0 & \\
\hline somex al & 'trust' & 67.3 & \\
\hline sone & ‘hate' & 77.6 & \\
\hline Soxe'ax & 'forget' & 88.0 & \\
\hline
\end{tabular}

Table 3: preference for singular objects in Experiment 3 


\subsection{Experiment 4 - preferences of reciprocal interpretations (text-based)}

Method 103 participants (76 male and 27 female, average age 24) received one of two forms, which together contained 69 questions 4 Of the 69 questions, 16 were test items and 53 were fillers. In each test item participants were presented with a reciprocal sentence containing a subject referring to three people. The participants were asked whether it is necessary or not to conclude from the given sentence that one person of those referred to acted on another one (both persons were given by name). The predicates tested were the same ones as in Experiment 3. As in Experiment 3, each "eventive" predicate was tested once in the perfective aspect and once in the imperfective, with at least three other test items separating between the two test items for the predicate. Sentences (8) and (9) illustrate these test items, where (10) is the question asked.

(8) baSana Se'avra omer, boaz ve-gai dakru exad-et-haSeni in-the-year that-passed Omer, Boaz and-Guy stabbed one another

"Last year, Omer, Boaz and Guy stabbed one another."

Rina nixnesa ve-hivxina be'omer, boaz ve-gai dokrim

Rina entered and-noticed in-Omer, Boaz and-Guy stabbing

exad-et-hasheni

one another

"Rina entered the room and noticed Omer, Boaz and Guy stabbing one another."

(10) ha'im nitan lehasik mi-kax she-gai dakar et boaz is-it possible deduce from-this that-Guy stabbed ACC Boaz

"Can you deduce from this that Guy stabbed Boaz?"

For each test item we collected the percentage of participants who answered negatively. A negative answer indicates that the reciprocal sentence can be interpreted as true in a situation where not all pairs between the three people appear in the relation denoted by the verb. We hence classify a negative answer as preference for a weaker reciprocal interpretation. A positive response to the question indicates a strong reciprocity interpretation, where the denoted relation includes all pairs between the different elements of the subject. The fillers contained sentences involving three people in various configurations and a different question about the situation than the question of the test items.

Results Table 4 shows the predicates tested and for each predicate, the percentage of participants who preferred the weaker reciprocal interpretation over the strong one. Due to a typo that was discovered in the questionnaire with the verb no'em ('give a speech'), this item was excluded from the analysis.

\footnotetext{
${ }^{4}$ Experiment 4 required a larger number of fillers than the other experiments, which made it necessary to have twice as many participants, and two different forms of the questionnaire.
} 


\begin{tabular}{|c|c|c|c|}
\hline Predicate & Translation & \multicolumn{2}{|c|}{$\begin{array}{l}\text { Preference of weaker reciprocal interpretation (percent) } \\
\text { perfective } \quad \text { imperfective }\end{array}$} \\
\hline ro'e & 'see' & 0 & 3.9 \\
\hline makSiv & 'listen to' & 6.3 & 8.2 \\
\hline maxmi & 'compliment' & 12.0 & 14.3 \\
\hline doker & 'stab’ & 16.0 & 31.3 \\
\hline soret & 'scrape' & 19.2 & 28.9 \\
\hline macbi'a & 'point at' & 25.0 & 11.8 \\
\hline somex al & 'trust' & 4.0 & \\
\hline Soxe'ax & 'forget' & 4.1 & \\
\hline mefake'ax & 'supervise' & 13.7 & \\
\hline sone & 'hate' & 19.6 & \\
\hline
\end{tabular}

Table 4: preference for weaker reciprocal interpretations in Experiment 4

\section{Discussion}

Experiments 1 and 3 both test preference of different verbs for different patient cardinalities. Experiment 1 uses forced choice between pictures illustrating a verb concept with one or two patients. Such forced choice experiments are standard when testing other typicality preferences (e.g. Southgate and Meints 2001). Experiment 3 used sentence completion in a task of forced choice between a single patient and multiple patients. Both experiments show that there is a significant variability among transitive verbs with respect to patient cardinality. With a $p$-value of 0.05 and 53 subjects, 20 of the 32 verbs tested in Experiment 1 showed a preference for patient cardinality that is significantly different than chance: 4 verbs towards multiple patients and 16 towards a single patient. The other 12 verbs did not show any significant preference for patient cardinality. This experiment shows clearly that some verbs like 'point at', 'shake', 'hit' and 'stab', have a clear preference for one patient per agent. By contrast, other verbs - 'give a speech', and to a lesser extent 'photograph', 'draw' and 'hug' - have a preference for situations with two patients per agent. This is in agreement with common experience, where the former predicate concepts normally require a physical act directed to one location in space. By contrast, the latter concepts reasonably do not invoke this requirement. In the case of the predicate 'give a speech', a speech directed to one person is furthermore quite unlikely. Considerable consistency in patient cardinality judgements was found with the four predicates that were included in both Experiment 1 and Experiment 3: 'point at', 'stab', 'scrape' and 'give a speech' ${ }^{5}$ In both experiments, the verbs 'point at', 'stab' and 'scrape' all displayed significant preference for a single patient, whereas 'give a speech' displayed a strong preference for two patients.

As said above, the MTH expects correlations between preferences of one patient per agent in instances of binary predicate concepts, and acceptances of "weaker" reciprocal interpretations with those concepts. Experiments 2 and 4 test for acceptability of strong reciprocal interpretations (full graphs) vis à vis weaker

\footnotetext{
${ }^{5}$ Striving to test as many different predicates as possible, we only chose four predicates to be included in both experiments.
} 
interpretations. Due to the similar experimental settings of Experiments 1 and 2 (picture-based settings) and Experiments 3 and 4 (text-based settings), we expect the stronger typicality-reciprocity correlations to hold of those pairs of experiments.

Experiment 2 tested preferences for reciprocal interpretations, in pairs of situations contrasting two kinds of configurations:

(a) Circular configurations (three pairs in the relation) vs. linear path configurations (two pairs in the relation).

(b) Complete configurations (six pairs in the relation) vs. circular configurations.

The results of the (a) test items show for all verbs a clear preference (94-100\%) of reciprocal interpretations for the circular configuration over the path configuration. On their own, these results may be expected by the SMH: although both the path configurations and the circular configurations are not the maximal situations possible for the predicates tested, it may be supposed that participants, upon being forced to choose between them, choose the circular configuration, which is closer to the maximal configuration. The MTH also expects the preference of the circular configuration, since there is no reason we know to consider the path configurations as more typical for these verb concepts than the circular configurations. Thus, the circular configurations exhibit reciprocity according to the MTH, or at least: they are closer than the path configurations to a situation that exhibits reciprocity. In the (b) tests the results are quite different. All the (b) tests showed much lower preferences (34-76\%) for the situation containing more pairs, compared to the (a) tests. This fact is not expected by the SMH: here, unlike the (a) tests, the circular configurations are visibly not the maximal configurations possible. Hence, the significant appearance of preferences for such situations as exhibiting reciprocity cannot be explained by the SMH alone. This leads to one of our main empirical conclusions:

No Maximality: Situations supporting reciprocal sentences do not necessarily involve the maximal number of pairs possible in the relation denoted by the predicate antecedent of the reciprocal.

As discussed in Section 4, the MTH, unlike the $\mathrm{SMH}$, expects reciprocity to be possible with non-maximal relations, provided that these relations are maximally typical of the predicate concept. Thus, the MTH expects a correlation between preferring circular configurations to complete ones in Experiment 2, and typicality of a single patient with the relative concepts in Experiment 1. Figure 4 shows the correlation calculated for all verbs in Experiments 1 and 2 except the verb no'em ('give a speech' see discussion below). The analysis (cf. Figure 4) shows a strong positive correlation (at +0.37 ), which is however statistically insignificant (Pearson correlation coefficient of 0.4 ). We attribute this to the poor distribution of values for patient cardinality typicality with the predicates we selected: all the predicates except 'give a speech' and 'hug' showed relatively strong preference for single patient interpretation. Although not statistically significant, the strong positive correlation leads us to expect that with a better distribution of the verbs across preferences for patient typicality, a more significant correlation may appear. 


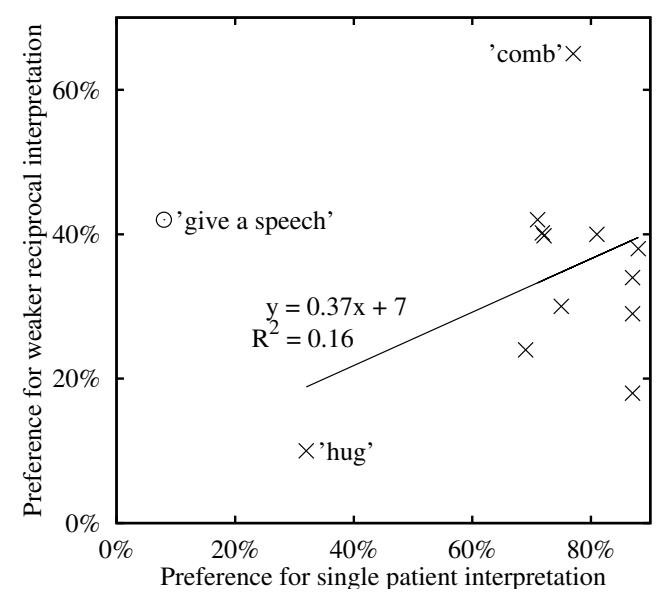

Figure 4: Experiments 1 and 2 - correlation between preference of circular configuration over complete configuration (weaker reciprocal interpretation) in Experiment 2, and preference of single patient in Experiment 1

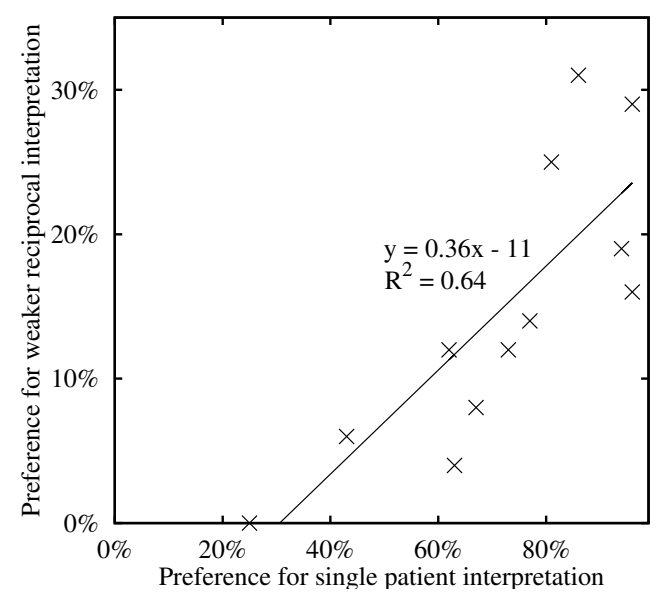

Figure 5: Experiments 3 and 4, six eventive predicates in the perfective and imperfective - correlation between acceptance of weaker reciprocity in Experiment 4 , and preference of a singular object in Experiment 3

Moving on to Experiments 3 and 4, the MTH again expects a correlation between preference of a singular object in Experiment 3 and acceptance of the weaker interpretation of the reciprocal as tested in Experiment 4. We analyzed the correlation between the six eventive predicates that were tested in these experiments separately from the stative predicates. With the two aspects of each eventive predicate, the calculated correlation is given in Figure 5. In this case there is again a strong positive correlation, similar (at +0.36$)$ to the correlation found with Experiments 1 and 2. Furthermore, in this case the correlation is statistically significant, with a Pearson correlation coefficient of 0.8 . Thus, among eventive predicates a large portion of the variance in the acceptance of weaker reciprocal interpretations may be attributed to patient cardinality preferences, as expected by the MTH.

On top of this quantitative correlation between Experiments 3 and 4, they also show a qualitative correlation between changes in typicality/reciprocity preferences when comparing the perfective and the imperfective aspects of the same eventive verb. Regarding the eventive verbs in Table 3, the imperfective aspect shows an average increase of $0.9(15 \%)$ in the preference of a single patient over the preferences observed in the perfective aspect. In correlation to that increase, the imperfective aspects shows an average increase of $0.03(25 \%)$ in the acceptance of weaker reciprocal interpretations, compared to the perfective aspects. All individual eventive predicates except the verb doker ('stab') also show a qualitative correlation in the direction of the change of preferences between imperfective and perfective aspects. We consider these correlations as further support for the MTH:

Typicality affects non-maximality: One of the factors affecting non-maximality of the relation denoted by a predicate antecedent in a reciprocal sentence is this 
predicate's typicality preference for single patients.

Apart from this evidence, which we consider to be supporting the MTH, the stative predicates tested in Experiments 3 and 4 did not show any support for our hypotheses. No correlation was observed between typicality preferences and reciprocal interpretations with the four stative verbs in these experiments. We believe that more research is needed about the factors that determine weaker interpretations of reciprocity with such verbs.

The behavior of another verb, no'em ('give a speech'), challenges our hypotheses most dramatically. While in Experiment 1, this verb showed the strongest preference for two patients $(92.5 \%)$, in Experiment 2 this verb did not show significant reciprocity preference for either of the circular/complete configurations. We speculate, however, that this should not be construed as counter evidence to the MTH. In fact, it is quite possible that 'give a speech' also shows a preference for agent cardinality and not only patient cardinality. Just like it is atypical to give a speech to one person, it is also quite atypical to be given two speeches simultaneously. As a result, the typicality of complete configurations (cf. Figure 6(a)) may in fact be lower than the typicality of circular configurations (cf. Figure 6(b)). If this is the case, preferences for patient cardinality in situations with one agent, in opposition to our tentative experimental assumption, is not indicative of the typicality of such situations where more agents and patients are involved. In such cases, agent cardinality may also affect the interpretation of the reciprocal according to the MTH. We leave it for further research to study the effects on reciprocal interpretation in such cases.

(a)

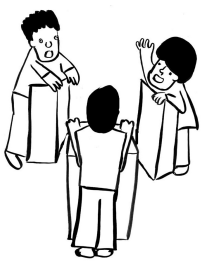

(b)

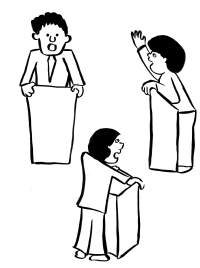

Figure 6: two situations illustrating the expression giving a speech to each other

\section{Conclusions}

We proposed the Maximal Typicality Hypothesis, a modification of the Strongest Meaning Hypothesis, as a new principle for analyzing the interpretation of reciprocal sentences. Following Sabato and Winter (2005), we proposed that the interpretation of reciprocals is directly derived by properties of the concept denoted by the binary predicate to which the reciprocal attaches. Thus, we implemented the MTH as formally generalizing the SMH, but employed a richer theory of predicate concepts than the "classical" theory of concepts presupposed by the SMH. Specifically, the typicality preferences of binary predicate concepts are hypothesized to play a major role in the logical interpretation of reciprocals. In order to check this 
hypothesis, we conducted a set of experiments involving reciprocal interpretations and typicality preferences with binary predicates. The correlations found between these different phenomena are claimed to support the MTH. Almost inevitably, the complexities of semantic judgements about reciprocals require further theoretical and experimental work. We believe however, that our main proposals and findings may point to some general connections between conceptual structure and the logical behavior of natural language expressions.

\section{References}

Armstrong, S., Gleitman L., and H. Gleitman: 1983, 'What some concepts might not be', Cognition 13, 263-308.

Dalrymple, M., M. Kanazawa, Y. Kim, S. McHombo, and S. Peters: 1998, 'Reciprocal Expressions and the Concept of Reciprocity', Linguistics and Philosophy 21, 159-210.

Lakoff, G.: 1973, 'Hedges: A study in meaning criteria and the logic of fuzzy concepts', Journal of philosophical logic 2, 458-508.

Margolis, E. and S. Laurence: 1999, Concepts: Core Readings. MIT Press.

Osherson, D. N.: 1990, 'Category-based induction.', Psychological review 97, 185200.

Osherson, D. N. and E. E. Smith: 1997, 'On Typicality and Vagueness', Cognition 64, 189-206.

Rosch, E.: 1975, 'Cognitive representations of semantic categories', Journal of Experimental Psychology: General 104, 192-233.

Rosch, E.: 1976, 'Structural Bases of Typicality Effects.', Journal of Experimental Psychology: Human Perception and Performance 2, 491-502.

Rosch, E. and C. B. Mervis: 1975, 'Family Resemblances: Studies in the Internal Structure of Categories.', Cognitive Psychology 7, 573-605.

Sabato, S. and Y. Winter: 2005, 'From Semantic Restrictions to Reciprocal Meanings', Proceedings of FG-MoL.

Smith, E. E. and D. L. Medin: 1981, Categories and Concepts. Harvard University Press.

Southgate, V. and K. Meints: 2001, 'Typicality, naming, and category membership in young children', Cognitive Linguistics 11, 5-16.

Winter, Y.: 2001, 'Plural predication and the Strongest Meaning Hypothesis', Journal of Semantics 18, 333-365. 